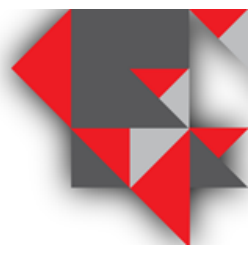

\title{
La negociación de las reglas de origen en el T-MEC, implicaciones para su puesta en práctica
}

\author{
Raúl Gustavo Acua Popocatl ${ }^{1}$, Selene Jiménez Bautista ${ }^{2}$ y Darío Guadalupe Ibarra Zavala ${ }^{3}$ \\ ${ }^{1}$ Profesor-investigador de la Licenciatura en Comercio Internacional, Centro Universitario Nezahualcóyotl, \\ Universidad Autónoma del Estado de México, miembro del cuerpo académico "Comercio, economía y negocios \\ internacionales para el desarrollo”. acuagus@yahoo.com \\ ${ }^{2}$ Profesora-investigadora de la Licenciatura en Comercio Internacional, Centro Universitario Nezahualcóyotl, \\ Universidad Autónoma del Estado de México, líder del cuerpo académico "Comercio, economía y negocios \\ internacionales para el desarrollo”.sejiba22@gmail \\ 3 Profesor-investigador de la Licenciatura en Comercio Internacional, Centro Universitario Nezahualcóyotl, \\ Universidad Autónoma del Estado de México, miembro del cuerpo académico "Comercio, economía y negocios \\ internacionales para el desarrollo”. darioibarra@yahoo.com
}

\author{
Información del artículo revisado por pares \\ Fecha de aceptación: junio-2021 \\ Fecha de publicación en línea: diciembre-2021 \\ DOI: https://doi.org/10.29105/vtga7.2-43
}

\section{Resumen}

En agosto de 2017 comenzaron las negociaciones para la modernización del Tratado de Libre Comercio de América del Norte, en el tema de la actualización de las reglas de origen, el gobierno de los Estados Unidos aplicó una estrategia distributiva de regateo para imponer a México y Canadá una redacción de las reglas acorde con su objetivo de contribuir con ellas a atenuar su desequilibrio comercial con sus socios, pero se tuvieron que hacer concesiones por las tres partes para la simplificación de los mecanismos para la aplicación práctica de tales reglas.

Palabras clave: T-MEC, reglas de origen, negociaciones comerciales internacionales, facilitación comercio

\section{INTRODUCCIÓN}

El objetivo del presente estudio consiste en comprender el proceso de negociación realizado por los funcionarios del gobierno de México, Estados Unidos y Canadá que llevó a la modificación de las reglas de origen contenidas en el TLCAN para plasmarlas en el nuevo T-MEC, se procura explicar los factores que influyeron para obtener normas inferiores a las óptimas que estaban disponibles en la mesa de negociación, para ello se analizarán los siguientes elementos: la importancia teórica y

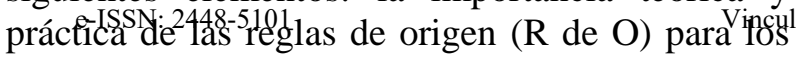

Abstract

In August 2017, negotiations took place for updating the North American Free Trade Agreement, regarding the reform of the rules of origin, the United States government resorted to a distributive bargaining strategy to impose on Mexico and Canada a drafting of the rules in accordance with its goal of alleviating its trade imbalance with its trade partners, but concessions had to be made by the three countries in the simplification of the mechanisms for their application.

Keywords: USMCA, rules of origin, international trade negotiations, trade facilitation.

intercambios negociados en las áreas de libre comercio, las características básicas contenidas en estas reglas que se plasmaron en el TLCAN, las 3 opciones sugeridas por los especialistas para hacer más eficientes las reglas en el nuevo T-MEC, los intereses y las propuestas de México en la negociación, los intereses y propuestas de EU, los intereses y propuestas de Canadá y el resultado final plasmado en el nuevo T-MEC. Este estudio básicamente es uno comparativo en donde se contrastan las preferencias comerciales de negociación de los actores involucrados, como laTégicansumo para explicar el resultado finałol. 7 Núm. 1 Enero-Junio 2021 
Para comprender la importancia de este tipo de reglas primero es relevante saber que el tipo de comercio internacional que se presenta en estos umbrales del siglo XXI básicamente se caracteriza por que el $80 \%$ de las transacciones se procesan a través de las cadenas globales de valor que son gestionadas por las empresas transnacionales (UNCTAD, 2013), y por la existencia de un número significativo de normas comerciales negociadas entre los gobiernos de los países a diferentes niveles (multilateral, plurilateral, regional y bilateral), las cuales son actualizadas constantemente para estar a tono regulatorio, en la medida de lo posible, con esta realidad comercial siempre cambiante. Este tipo de transacciones comerciales han hecho que en la actualidad el tema principal de la negociación de los acuerdos comerciales internacionales ya no sea la desgravación arancelaria y el acceso a mercados para productos manufacturados o agrícolas, sino que su propósito ahora sea sentar las bases jurídicas de lo que se ha llamado como la economía regulatoria que abarca otros temas novedosos y prioritarios como los relacionados con la facilitación del comercio.

Esta economía regulatoria exige que la desagregación de la producción que caracteriza a los negocios internacionales actuales se sustente en una regulación de las nuevas actividades que tienen lugar entre los países, como la libre circulación de insumos, servicios y capitales internacionales, lo que se procura en la actualidad es eliminar las barreras que resultan burocráticamente onerosas y que inhiben la incorporación de nuevas empresas (como las pequeñas y medianas) en el comercio internacional (Vega, 2020). Entre las nuevas regulaciones que los mercados exigen para reducir los costos de transacción se hallan las R de $\mathrm{O}$.

Estas reglas son incluidas en los acuerdos de libre comercio regionales para que las partes se aseguren que los productos elaborados fuera del área de libre comercio (ALC) negociada, no se beneficien de las preferencias arancelarias y del acceso a mercado que reciben los productos elaborados regionalmente dentro de las propias ALC. Debido a que los países que forman parte de una ALC tienen diferentes aranceles de Nación Más Favorecida (NMF) aplicados a los bienes producidos externamente ( $\sin \mathrm{R}$ de $\mathrm{O}$ ), la creación de una ALC que no cuente con estas reglas podría propiciar un marco desleal en donde las importaciones fuera del ALC evadirían los aranceles al ser vendidas al país con el arancel más bajo, y luego se reexportasen a otro miembro del área de libre comercio.
En relación con el comercio en América del Norte, el cual se hallaba regulado por el Tratado de Libre Comercio (TLCAN) firmado por México, Estados Unidos y Canadá en 1994, en agosto de 2017 se decidió modernizarlo como consecuencia de las exigencias del entonces nuevo presidente de los Estados Unidos el republicano Donald Trump. La reapertura del TLCAN abarcaría la renegociación de las $\mathrm{R}$ de $\mathrm{O}$ plasmadas en ese acuerdo. Tales reglas se consideraban muy estrictas, pues fueron una evolución de las que se decidió incorporar en el anterior CUSFTA (Canada-United States Free Trade Agrement) entre Canadá y los Estados Unidos que entró en vigor en 1988. Las $\mathrm{R}$ de $\mathrm{O}$ que se incorporaron en 1994 se caracterizaban porque variaban de un producto a otro, y normalmente requerían cuotas de valor regional más altas que las contenidas en otros acuerdos similares negociados en aquella época.

El desafío que se presentaba a los negociadores de los tres países en el 2017 era simplificar los procesos relacionados con la certificación de origen, mediante tres grandes opciones disponibles, la primera era la redacción de una regla uniforme que se aplicara a todos los productos, estableciendo un criterio de contenido regional simple. Una segunda opción (no excluyente con la primera) disponible era ampliar el umbral de minimis por debajo del cual los productos no tienen que pasar por una certificación de origen, sobre todo el aplicado a aquellos productos de bajo arancel, apoyando así a las pequeñas empresas para las cuales es complicado el cumplimiento de las $\mathrm{R}$ de $\mathrm{O}$. Por último, una acción adicional era avanzar hacia una norma global que facilitase a los exportadores la conformidad con las reglas de los diferentes acuerdos comerciales, y que también permitiese la acumulación de origen.

La exploración, el análisis, la presentación formal y discusión de estas tres propuestas para modernizar y al mismo tiempo hacer más eficientes las $\mathrm{R}$ de $\mathrm{O}$ del TLCAN, sin embargo, iba a estar obstaculizada por los intereses económicos y particularmente los intereses políticos de los representantes de los gobiernos involucrados, así como por sus estrategias y tácticas de negociación distributivas aplicadas durante las rondas que duraron de agosto de 2017 a diciembre de 2018, lo cual impactó el resultado final obteniendo resultados menores a los óptimos de todos los que se discutieron en la mesa.

El tipo de negociación que se puso en evidencia en las $\mathrm{R}$ de $\mathrm{O}$ pareció ser de carácter distributivo en donde los Estados Unidos impusieron sus opciones a 
Canadá y México que se mostraron más a la defensiva procurando mantener el status quo en las reglas, por encima de las demandas de EU para endurecerlas de tal forma que, según sus argumentos, se pudiera superar el desequilibrio en la balanza comercial de ese país. Durante la renegociación del TLCAN, la política interna de EU empujó al gobierno de Trump para buscar reglas más estrictas, sobre todo aquellas que se aplicaban a los productos más sensibles desde el punto de vista tanto económico como político.

\section{MARCO TEÓRICO}

Varios han sido los enfoques teóricos que han procurado hallar los motivos que tienen los gobiernos de los países para dejar atrás conductas económicas-comerciales egoístas y unilaterales, para echar a andar negociaciones con el fin de alcanzar acuerdos de cooperación económica profunda como sucede con las ALCs que son por lo general acuerdos alcanzados entre países para eliminar por completo los aranceles entre ellos, éstas ALC han comenzado a tener un auge impresionante a partir de mediados de los ochenta, al grado que en el año 2020 la OMC registró 306 acuerdos de este tipo creados entre sus miembros, aunque no todos son auténticamente áreas de libre comercio $^{1}$. Las ALC son arreglos regionales o bilaterales que se diferencian de otros acuerdos porque su membresía abarca a un número reducido de países vinculados geográficamente, o ligados por intercambios comerciales más relevantes entre ellos, en relación con el resto de los países que forman el sistema comercial internacional.

Desde el punto de vista teórico, el primer efecto percibido que tiene lugar después de la creación de un ALC es la expansión de los flujos de comercio entre los países fundadores de ellas, esto se suscita frecuentemente a costa del comercio que se tiene con países que no son miembros del ALC, lo cual es una problemática frecuente en estos arreglos. Esta expansión, que es una consecuencia de la eliminación de las barreras arancelarias, puede producir un efecto creador de comercio lo que significaría que los proveedores más eficientes en los socios que integran el ALC reemplazarían a los proveedores nacionales de una mercancía determinada, pero también se presentarían otros efectos conocidos como incitadores de la desviación del comercio, que significa que los

${ }^{1}$ https://www.wto.org/english/tratop_e/region_e/region_ e.htm\#: : :text=As $\% 20$ of $\% 2020 \% 20$ September $\% 202020$ ,the $\% 20$ GATT $\% 20$ or $\% 20$ the $\% 20$ WTO $\% 3 \mathrm{~F}$ proveedores externos más eficientes son desplazados por los menos eficientes dentro del ALC, como resultado de la liberalización discriminatoria. En el momento en que la desviación del comercio domina a la creación del comercio, se argumenta que las ALC tienden a reducir el bienestar. Los niveles de desvío del comercio están estrechamente relacionados con el grado de discriminación establecido en el acuerdo, es por esto que las $\mathrm{R}$ de $\mathrm{O}$ buscan precisamente que los efectos de creación predominen sobre aquellos que provocan la desviación del comercio (Viner, 1950).

En la medida en que crece el número de acuerdos de libre comercio, también lo hace el problema de sus diferentes $\mathrm{R}$ de $\mathrm{O}$. Para los productores, esta proliferación de reglas significa que sus productos deben ser fabricados y certificados de manera única para calificar y obtener las preferencias adquiridas en diferentes acuerdos de tipo ALC. Las diferencias en las desgravaciones arancelarias, a menudo sustanciales, obligan a las empresas a elegir entre las diferentes ALC a su disposición para aplicar las mejores preferencias a sus productos o insumos, generalmente las preferencias elegidas son aquellas que reducen ampliamente los costos de transacción internacional. La teoría económica muestra que las $\mathrm{R}$ de $\mathrm{O}$ pueden usarse para transferir la protección de un bien final a las partes y componentes usados para producirlo. Dado que el incumplimiento de las normas de origen descalifica a un exportador del trato preferencial conferido por un ALC específico, las reglas suelen ser el instrumento central de acceso al mercado. En particular, la relevancia de las reglas como "guardianes del comercio" puede acentuarse con el tiempo ya que estas reglas permanecen vigentes incluso después de que se hayan eliminado gradualmente los aranceles preferenciales (Moroz, 2017).

Las $\mathrm{R}$ de $\mathrm{O}$ fueron redactadas de cierta forma para crear una barrera de entrada para empresas nuevas o pequeñas, porque para poder tener acceso a las preferencias pactadas en las ALC se deben obtener los certificados de origen. Si bien las $\mathrm{R}$ de $\mathrm{O}$ alientan a los productores a utilizar insumos regionales, también es un hecho que aumentan los costos administrativos y de producción. Esto obliga a los exportadores a sopesar los costos de cumplir con las $\mathrm{R}$ de $\mathrm{O}$, con las preferencias arancelarias recibidas. Debido a que los grandes exportadores 
tienen oficinas administrativas más sofisticadas que pueden manejar eficientemente los costos de documentar el origen, esto les propicia una gran ventaja. En cambio, para las pequeñas empresas interesadas en ingresar al mercado de las exportaciones, los costos adicionales relacionados con documentar el origen pueden desalentar la exportación. Dado que el costo administrativo de las normas de origen actúa como un costo fijo, esto impone una carga más pesada a las pequeñas empresas y a los productos de bajo valor.

Se debe tomar en cuenta que existen dos tipos de $\mathrm{R}$ de $\mathrm{O}$, las normas de origen no preferenciales $\mathrm{y}$ las preferenciales. Las primeras se utilizan para distinguir los productos extranjeros de los nacionales al establecer los derechos antidumping y compensatorios, las medidas de salvaguardia, los requisitos de origen y/o las restricciones cuantitativas discriminatorias o contingentes arancelarios, así como en los casos de contratación pública. Las $\mathrm{R}$ de $\mathrm{O}$ preferencial definen las condiciones bajo las cuales el país importador va a considerar que un producto es originario de un país exportador elegible para un trato preferencial del país importador. Las ALC utilizan las reglas para determinar si una mercancía califica para un trato preferencial cuando se exporta de un estado miembro de la ALC a otro (WTO, 2018).

Además de las $\mathrm{R}$ de $\mathrm{O}$ específicas, existen también a) las normas de minimis, b) el llamado principio de acumulación, y c) el tipo o modalidad de acumulación, que son criterios importantes para entender a las $\mathrm{R}$ de $\mathrm{O}$ y su utilidad en el comercio internacional. La mayoría de las ALC contienen algún tipo de regla de minimis que permite utilizar un porcentaje máximo especificado de materiales no originarios que no afectan el origen. La regla de minimis tiene como fin flexibilizar los criterios aplicados en los cambios de la clasificación arancelaria -o en los requisitos técnicos- mediante la facilitación de la calificación de los productos

\footnotetext{
2 "El principio de absorción (roll-up) prevé que una vez que el producto haya obtenido el carácter de originario, todas las materias no originarias utilizadas en su fabricación se considerarán como originarias, y las disposiciones de mayor o menor complejidad sobre acumulación". Estudio Comparativo sobre las Normas de Origen Preferenciales. (2018). Consultado en http://www.wcoomd.org/-

/media/wco/public/es/pdf/topics/origin/instruments-andtools/reference-material/170130-c-_sp-comparativestudy-on-pref_roo_-master-file_final-23_06_2017.pdf

3 "El concepto de "acumulación", llamado también "normas de origen acumulativas" permite que los países
}

con insumos que son no originarios. El principio de acumulación, por otra parte, permite que los materiales que han adquirido origen al cumplir requisitos específicos de procesamiento se consideren originarios cuando se utilizan como insumos en una transformación posterior. Cuando se permite el llamado roll-up ${ }^{2}$, los materiales no originarios no se tienen en cuenta en el cálculo del valor agregado de la transformación posterior.

La posibilidad de que la determinación de origen de un bien tome en cuenta la producción realizada por más de una empresa -o en más de un país- es conocida como acumulación. La acumulación permite a los productores de un país miembro de un ALC utilizar materiales de otro miembro dentro del ALC (u otros miembros) sin perder el estatus preferencial del producto final. Existen 3 tipos de acumulación ${ }^{3}$. La acumulación bilateral que tiene lugar entre los dos socios dentro del ALC y que les permite utilizar productos que se originan en el otro socio del ALC como si fueran propios cuando buscan calificar para el trato preferencial relacionado con el ALC en ese socio. Prácticamente todos los regímenes basados en $\mathrm{R}$ de O aplican la acumulación bilateral. En la llamada acumulación diagonal, los países vinculados por el mismo conjunto de $\mathrm{R}$ de $\mathrm{O}$ preferenciales pueden utilizar productos que se originan en cualquier parte del régimen aplicado como si fueran originarios del país exportador. Por su parte, la acumulación total extiende la acumulación diagonal. Establece que los países vinculados por el mismo régimen de $\mathrm{R}$ de $\mathrm{O}$ tienen derecho a utilizar bienes producidos en cualquier parte de la zona común, esto significa que en general el procesamiento realizado en la zona se calcula como si hubiera tenido lugar en el país de fabricación final.

En resumen, la acumulación diagonal y total pueden contribuir a expandir significativamente el alcance geográfico y el número de productos de un régimen basado en $\mathrm{R}$ de $\mathrm{O}$, mientras que las reglas

que forman parte de un régimen comercial preferencial puedan compartir la producción y satisfacer en conjunto las disposiciones pertinentes sobre las normas de origen. Dicho de otro modo, la acumulación permite que los productos de un país perteneciente a un régimen comercial preferencial sean objeto de elaboraciones o transformaciones en otra parte de este mismo régimen comercial preferencial, como si fueran originarios de esta última parte. De esta manera, la producción puede verse complementada con los insumos procedentes de otros países sin perder su carácter originario, y de esta manera, se brindan nuevas oportunidades para proveerse de insumos". Ibidem. 
de minimis, roll-up y acumulación permiten cierta flexibilidad en la aplicación de las reglas. Cuando las $\mathrm{R}$ de $\mathrm{O}$ se redactan de forma muy estricta como ocurrió en el TLCAN, éstas obligan a aumentar la participación o el nivel de valor agregado que debe producirse regionalmente, es pertinente subrayar que la protección originalmente concebida para los bienes finales también se transfiere a los suministros que se utilizan para producirlos. Esta modalidad de protección significa que algunas piezas necesarias para la producción regional sean las que se utilicen, aún a pesar de que los componentes extranjeros sean más baratos.

Subrayemos que el tipo de transacciones comerciales presentes en el siglo XXI son una modalidad evolucionada de la desagregación de la producción, en donde las cadenas globales de valor y de abasto, así como las políticas de liberalización unilateral aplicadas por algunos países como México, requieren un nuevo tipo de acuerdos centrados básicamente en la economía regulatoria en la cual temas como la facilitación del comercio resultan más importantes que la propia desgravación y el acceso a mercados, por ello es importante poner atención a las $\mathrm{R}$ de $\mathrm{O}$ y su formulación, porque su contenido pueden contribuir a acelerar -o por el contrario a inhibireste tipo de desagregación productiva.

A lo anterior debe sumarse que la propia Organización Mundial del Comercio impone pocas disciplinas estrictas sobre las $\mathrm{R}$ de $\mathrm{O}$, como resultado de esto, las reglas se han utilizado no solo para que las partes en un ALC se protejan legítimamente contra el abuso de la triangulación y el transbordo de productos, sino como una forma de protección comercial, particularmente en productos sensibles como la industria del vestido, el calzado, los textiles, los alimentos y los automóviles (Lipsey, 1992).

\section{METODO}

El método aplicado en este estudio es de naturaleza comparativa en donde se contrastan las preferencias de negociación -y las estrategias de regateoreveladas a los medios de comunicación por los funcionarios de los tres países que se encargaron de negociar las $\mathrm{R}$ de $\mathrm{O}$ en el T-MEC durante el período 2017-2019. En el ámbito jurídico, metodológicamente existen dos vías para determinar el nivel de eficiencia que las $\mathrm{R}$ de $\mathrm{O}$ aportan al comercio regional en las ALC, la primera es la determinación de los costos relacionados con la aplicación práctica de las $\mathrm{R}$ de $\mathrm{O}$, los cuales se pueden obtener indirectamente a través de un mecanismo de preferencia revelada, recurriendo a estadísticas sobre las tasas de utilización del TLCAN proporcionados, entre otras fuentes, por la Comisión de Comercio de los Estados Unidos. La opción que enfrentaban, por ejemplo, los exportadores mexicanos era enviar mercancías a EU o Canadá bajo el TLCAN, lo que les daba derecho a ciertas preferencias arancelarias pero ser elegible para ellas les requería el cumplimiento de las reglas del TLCAN, en particular las R de O, o exportarlas bajo arancel de NMF con reglas de la OMC, lo que los obligaba a pagar el arancel NMF comprometido con los EU, esto no requería el cumplimiento con las reglas del TLCAN. De esta forma, en sectores donde la tasa de utilización del TLCAN es del $100 \%$, el beneficio de la preferencia arancelaria se presentaba como mejor que optar por los costos de cumplimiento. Por lo tanto, en esos sectores, la tasa de preferencia arancelaria aporta un límite superior al equivalente ad valorem de los costos de cumplimiento.

En cambio, en los sectores donde la tasa de utilización del TLCAN es cero, si aplicamos el mismo criterio, la preferencia arancelaria aporta un límite inferior a los costos de cumplimiento. Finalmente, en todos los sectores donde las tasas de utilización se encuentran estrictamente entre cero y $100 \%$, si los exportadores mexicanos tuvieran costos de cumplimiento simétricos, se revelarían indiferentes entre la exportación bajo reglas del TLCAN o pagando aranceles NMF, por lo que la tasa de preferencia arancelaria se revelaría igual a los costos de cumplimiento. Ante la disponibilidad de costos de cumplimiento heterogéneos, lo que se puede decir es que la preferencia arancelaria aporta una estimación aproximada de los costos de cumplimiento en el sentido de que al menos algunas empresas tienen costos de cumplimiento más altos mientras que otras tienen costos más bajos (Senger, 2018).

Lo anterior nos permite comprender que en el TLCAN, el nivel de complejidad de las reglas tuvo un impacto negativo en el recurso a las preferencias comerciales por parte de las empresas mexicanas debido a que el cumplimiento de los requisitos se convirtió en un trámite costoso que les quitaba mucho tiempo. Existe el dato de que de 3,500 productos mexicanos importados por Estados Unidos, en promedio, el $20 \%$ entra en ese país sin tomar ventaja de las preferencias arancelarias a pesar de ser elegibles, debido a la complejidad de las $\mathrm{R}$ de O. A eso hay que añadir que en México se calcula que en cada transacción aduanera promedio 
intervienen entre veinte y treinta partes diferentes, se ocupan cuarenta documentos y al menos doscientos tipos de datos, lo que eleva los costos de transacción aún más (OMC, 2014). Estos costos administrativos adicionales implican que algunos empresarios opten por pagar los aranceles de NMF de la OMC, en lugar de aprovechar las preferencias comerciales del TLCAN. También significa que algunas empresas terminaron por decidir no participar en el comercio del TLCAN, en particular las pequeñas empresas que carecen del personal legal con conocimientos sobre el proceso de otorgamiento de certificados de origen del propio TLCAN.

El segundo método para calcular la eficiencia de las $\mathrm{R}$ de $\mathrm{O}$ lo desarrollaron Estevadeordal y Suominen en un estudio en el que examinaron las $\mathrm{R}$ de $\mathrm{O}$ en 35 ALCs (Estevadeordal, 2002). Después de jerarquizar la restricción de cada regla en una escala del 1 al 7, promediaron las puntuaciones de los productos cubiertos por tales reglas. Hallaron que el TLCAN tenía las reglas más estrictas de todos los acuerdos, con un puntaje promedio de 5.1. En comparación, el acuerdo UE-México tiene una puntuación media de 4.8 y el ALC Japón-Singapur 4.6. Una razón por la que las reglas del TLCAN tendían a ser más onerosas era porque requerían transformaciones más extensas por cada categoría arancelaria. La transformación sustancial realizada bajo las reglas del TLCAN a menudo exigía un cambio de capítulo del propio acuerdo, y una clasificación más amplia de los bienes. Por el contrario, las reglas de la UE que requieren cambios de clasificación arancelaria se encuentran generalmente en el título de clasificación (es decir, en primeros cuatro dígitos de la clasificación arancelaria). Esto significa que las normas de la UE permiten que algunos productos del mismo capítulo se transformen, y continúen calificando para las preferencias, mientras que el TLCAN no lo permitía.

\section{RESULTADOS}

Quienes negociaron las $\mathrm{R}$ de $\mathrm{O}$ en el TLCAN procuraron hacerlas detalladas y acompañadas de sus respectivos procedimientos aduaneros, esto se debió a las experiencias negativas que la gestión de las mismas suscitó entre EU y Canadá cuando establecieron el CUSFTA en 1988, con base en esa experiencia los negociadores del TLCAN establecieron regulaciones uniformes para desarrollarse e instrumentarse conjuntamente por cada una de las tres partes del TLCAN. Así fue como se decidió que el Capítulo 4 del TLCAN describiese los requisitos de las $\mathrm{R}$ de $\mathrm{O}$. Los pormenores más importantes se describían en el Anexo 401 que abarcaba 304 páginas del TLCAN, el cual detallaba las $\mathrm{R}$ de $\mathrm{O}$ producto por producto. Las reglas del TLCAN requerían que las mercancías se originaran en la región o se transformaran significativamente en ella; aquellos bienes que se transformaban significativamente, es decir, que eran producidos con componentes de otros países, necesitaban un cambio en la clasificación arancelaria y / o debían cumplir con un porcentaje de contenido regional. Para ser elegible para las preferencias del TLCAN, el exportador debía completar una certificación de origen del TLCAN y entregarla al importador (Spak, 2018).

Acumulación. El artículo 404 establecía que para determinar si una mercancía calificaba como originaria, el exportador o productor de la mercancía podía "acumular" la producción de la mercancía de otros productores, siempre que sobre la base acumulada, todos los materiales no originarios se sometieran al cambio de clasificación arancelaria requerido, y que la mercancía lograra cumplir todo requisito de contenido de valor regional (RVC por sus siglas en inglés) aplicable. Este artículo permitía, por ejemplo, que una mercancía que el productor $\mathrm{A}$ procesó inicialmente en México y que el Productor B importó y procesó posteriormente en los Estados Unidos, calificaría como una mercancía originaria sobre la base de la producción total de los productores A y B, cuando el bien se exportara a Canadá.

De minimis. El artículo 405 del TLCAN establecía una excepción de minimis del 7\%. Esto significaba que el TLCAN consideraba un bien como originario si el valor de los materiales no originarios que no se sometían al cambio de clasificación arancelaria requerido era (a) "no más del 7\% del valor de transacción del bien, ajustado a una base de incoterm FOB, o, (b) si el valor de la transacción era inaceptable, no más del 7\% del costo total del bien. Un bien sujeto a un requisito de RVC calificaría para el trato preferencial si el valor de todos los materiales no originarios utilizados para producir el bien no era más del 7\% del valor de transacción ajustado.

Certificados de origen. El artículo 501 del TLCAN requería que las Partes emitiesen un Certificado de Origen para autenticar que una mercancía que se exportaba de una Parte a otra calificaba como mercancía originaria. Cada Parte podía exigir que el certificado de una mercancía importada se emitiera en un idioma requerido por sus leyes. En casos limitados, el TLCAN no requería un 
certificado, y la parte importadora podía renunciar al requisito de certificado en estas instancias. Un exportador debía completar y firmar un certificado para cualquier exportación de una mercancía para la que un importador podía reclamar un trato arancelario preferencial. Cuando el exportador no era el productor, el exportador podía completar y firmar el certificado considerando (a) el conocimiento del exportador si la mercancía calificaba como originaria; (b) la confianza razonable del exportador en la declaración escrita del productor de que la mercancía calificaba como originaria; o (c) la confianza del exportador en un certificado emitido y firmado para la mercancía que el productor proporcionó al exportador.

Tabla 1: Criterios en el TLCAN para las $R$ de $O$

\begin{tabular}{|c|c|}
\hline $\begin{array}{l}\text { Categorías } \\
\text { (tipos de } \\
\text { productos) }\end{array}$ & Descripción \\
\hline $\mathrm{A}$ & $\begin{array}{c}\text { Productos "totalmente elaborados u } \\
\text { obtenidos" en América del Norte: } \\
\text { No contiene material ajeno al TLCAN. } \\
\text { Ejemplos: minerales; hortalizas cosechadas; } \\
\text { animales vivos nacidos y criados; pescados, } \\
\text { desperdicios y desechos derivados de esa } \\
\text { producción. }\end{array}$ \\
\hline B & $\begin{array}{l}\text { Productos que contengan "insumos no } \\
\text { originarios", pero que cumplan las R de O del } \\
\text { Anexo } 401 \text {. } \\
\text { Ejemplos: panes, bollería, tartas, panes } \\
\text { hechos con harina no originaria } \\
\end{array}$ \\
\hline $\mathrm{C}$ & $\begin{array}{l}\text { Productos fabricados íntegramente en la } \\
\text { región del TLCAN a partir de insumos } \\
\text { originarios (que contienen materiales que no } \\
\text { pertenecen al TLCAN pero que cumplen la } \\
\text { regla de origen del TLCAN). } \\
\text { Ejemplo: prensas para producir vino }\end{array}$ \\
\hline $\bar{D}$ & $\begin{array}{l}\text { Productos sin ensamblar con clasificación } \\
\text { arancelaria de la misma categoría que sus } \\
\text { partes: } \\
\text { No cumplen con la regla de origen del Anexo } \\
\text { 401, pero contienen suficiente valor de } \\
\text { contenido regional de América del Norte. } \\
\text { También deben cumplir la regla de minimis. } \\
\text { Ejemplo: Un exportador de EU de aceite de } \\
\text { palma podría importar petróleo crudo de un } \\
\text { tercer país bajo la regla de minimis y } \\
\text { refinarlo en México para exportarlo como } \\
\text { producto norteamericano. }\end{array}$ \\
\hline f_Or & $\begin{array}{l}\text { U.S. Department of Commerce, Trade Information } \\
\text { "Rules of origin: Making the NAFTA origin } \\
\text { ination," tomado de: } \\
\text { ww.worldtraderef.com/WTR site/NAFTA/Rules_o } \\
\text { n.html, [consultado } 24 \text { de enero 2021]. }\end{array}$ \\
\hline
\end{tabular}

En el propio acuerdo se pactó que los bienes "cultivados, cosechados, totalmente producidos o sustancialmente transformados" en América del
Norte pertenecerían a cualquiera de las siguientes cuatro categorías: A, B, C o D. La categoría A abarcaba bienes "totalmente producidos u obtenidos" en América del Norte (minerales, hortalizas recolectadas, animales, pescado, productos manufacturados y chatarra). La categoría B, o "insumos no originarios" (como los productos de confitería, que posteriormente iban a procesarse dentro de los Estados Unidos) dependía de su clasificación arancelaria; la categoría $\mathrm{C}$ abarcaba todos los productos manufacturados de América del Norte a partir de insumos "originarios" de la región para su posterior procesamiento o venta en otros lugares; y la categoría D adoptaba una regla de origen de minimis para todos los productos, excepto los lácteos, el cacahuate, varios aceites (excepto los aceites tropicales, los aceites hidrogenados y la margarina), los cítricos y el azúcar (todos los cuales deben ser de producción nacional). De acuerdo con esta regla, como mucho el $7 \%$ del bien que circulaba libremente podía ser de un país no originario (para los productos de tabaco, esto se elevaba al 9\%).

Los productos de América del Norte (u "originarios") se distinguían de los materiales no norteamericanos ("no originarios") utilizando el método de valor del Artículo 402 o el método de costo. En este caso, el método del valor de transacción (primera fórmula), tomado del Código de Valoración en Aduana de 1979 del GATT, utilizaba el incoterm de precio del flete a bordo (FOB), los costos de transporte, las regalías sobre el bien y los ingresos de fabricación en el momento de la reventa. El valor del material no originario se deducía y se multiplicaba por 100 para obtener una proporción. Para el método de costo neto, el valor del material no originario se restaba del costo neto del productor, luego se multiplica por 100 para obtener una proporción.

Resulta importante notar para el caso de México que sus importaciones con socios comerciales que no pertenecen al TLCAN han sido pocas, constituyendo apenas $0.5 \%$ de las importaciones totales y $2.8 \%$ de las exportaciones, lo cual hace pensar a algunos analistas como Alicia Puyana que estas estadísticas muestran que los otros acuerdos comerciales firmados por México no siguieron como meta realmente la diversificación de los mercados, pues si se comparan con el comercio que se tiene con otros países no abarcados en acuerdos comerciales, éstos representarían el 22.7 y el 5.8\% de las importaciones y las exportaciones mexicanas respectivamente, contribuyendo a un déficit comercial significativo (Puyana, 2014). 
Tomando en cuenta el contenido de tales importaciones y sus países de origen, se percibe que un porcentaje significativo son insumos $y$ componentes importados transitoriamente para la exportación después de su procesamiento en México. A final de cuentas este flujo comercial es parte del comercio intra-firma de las organizaciones productivas involucradas en las cadenas de abasto y de valor, cuyo principal destino exportador son los EU. En este contexto ¿para qué necesita los acuerdos comerciales adicionales al TLCAN el gobierno de México? La respuesta parece ser que la estrategia es una de convergencia de tales acuerdos con el TLCAN, para acercar los aranceles acordados en cada uno de los TLC, con los que ya existen en los EUA para terceros países, resultando así una negociación mexicana con sus socios ajenos al TLCAN que pretende acercar los aranceles a los que ya se negociaron con los Estados Unidos, y con ello se crea una suerte de arancel externo común en el TLCAN que no se necesitó negociar con EU, debido a su estrategia de este país de no comprometerse con este tipo de acuerdos por motivos soberanos (acuerdos similares a los de una unión aduanera, que se caracteriza por que las partes integrantes de la misma tienen aranceles externos comunes).

Un asunto que vale la pena destacar es que dentro del TLCAN también se había pactado la revisión constante de las $\mathrm{R}$ de $\mathrm{O}$ a través de un comité trinacional, ya que se anticipaba que los cambios en los procesos productivos en las empresas localizadas en México, EU y Canadá iban a influir para que se presionara por una modificación futura de los términos negociados en las $\mathrm{R}$ de $\mathrm{O}$ creadas en el TLCAN, fue por eso que en este acuerdo se negoció el artículo 414 que establecía la creación de un comité llamado Grupo de Trabajo sobre R de O a través del cual las partes actualizan las $\mathrm{R}$ de $\mathrm{O}$ considerando las modificaciones que tienen lugar en los procesos productivos de América del norte. Este comité aprobó modificaciones en los años 2005, 2006, 2007, y 2012. (Leycegui, 2012)

Propuestas técnicas para hacer eficientes las reglas.

El desafío que se presentaba a los negociadores

\footnotetext{
4 “One potential way to improve market access for small businesses is to raise the de minimis threshold under which rules of origin do not bind. Currently, shipments under $\$ 2,500$ are not obliged to meet the NAFTA certification requirement. But even for small businesses this is not a commercially viable amount. One alternative would be to base the threshold not on
}

de los tres países en el 2018 era simplificar los procesos relacionados con la certificación de origen, mediante tres grandes opciones disponibles, una de ellas era la redacción de una norma uniforme que se aplicara a todos los productos, estableciendo una regla de contenido regional simple de, por citar un ejemplo, 40 a $50 \%$. Con esto se permitía, según los especialistas (Reinch, 2021), que las complejas restricciones de cambio de categoría arancelaria tuvieran que modificarse para permitir siempre mostrar el valor regional por encima de la cantidad requerida como alternativa, considerando que una regla de contenido regional simple es más transparente, al mismo tiempo que mantiene la transformación de la categoría arancelaria alternativa, ofrece también cierta flexibilidad a las empresas. Con requisitos de contenido regional para todos los bienes establecidos en un arancel, en lugar del tipo de reglas negociadas producto por producto en el TLCAN, éstos permitirían avanzar hacia una norma global lo cual es un objetivo importante para garantizar que las empresas que exportan a múltiples destinos, no se vean obligadas a elegir entre distintos acuerdos.

Una segunda opción disponible para los negociadores, aunque no excluyente con la primera, era ampliar el umbral de minimis por debajo del cual los productos no tienen que pasar por una certificación de origen, sobre todo aplicado a aquellos productos de bajo arancel, de esta forma se apoyaba a las pequeñas empresas que encuentran engorroso el cumplimiento de las $\mathrm{R}$ de $\mathrm{O}^{4}$. Por último, una acción adicional era avanzar hacia una norma global que facilitase a los exportadores la conformidad con las reglas de los diferentes acuerdos comerciales, y que también permita la acumulación cuando los acuerdos regionales se amplíen para incluir a otros países.

Desviarse de estas sugerencias mediante el endurecimiento de las normas de origen sugerido por el presidente Trump se anticipaba costoso para los consumidores e introduciría ineficiencias para las empresas. Una reforma realmente positiva del TLCAN debía simplificar las $\mathrm{R}$ de $\mathrm{O}$ y crear un umbral de contenido de valor regional único y más bajo de tal forma que se pueda aplicar de manera

the value of the goods shipped but on the value of the tariff revenue, with higher de minimis thresholds on lower tariff products..."

Fred Bergsten, (2019) A Path Forward for NAFTA. 2019, July 09. Consultado el 23 de noviembre 2020, en https://www.piie.com/publications/piie-briefings/pathforward-nafta, p. 121. 
uniforme a todos los productos. El gobierno del presidente Trump, se había presentado durante la campaña electoral como uno que favorecería la reducción de las regulaciones y no su aumento, esto habría anticipado que simplificaría los requisitos de las $\mathrm{R}$ de $\mathrm{O}$ en lugar de hacerlas más complejas.

\section{Intereses y posición de México}

En la presentación de los objetivos nacionales de México en las negociaciones del T-MEC hubo mucha retórica, e incluso la declaración pública de que "no se revelarían los intereses y objetivos del gobierno mexicano ante la opinión pública, para no debilitar su estrategia de negociación (sic)" se interpretaba más bien como una cortina de humo para no reconocer una definición de objetivos preliminar. Sin embargo, destacaba su propósito de mantener al país como plataforma de producción, exportación y captadora de la inversión de la región, lo cual reafirmaría un enfoque distributivo de la negociación en el entendido de que el resultado final debía beneficiar más a una de las partes que a las otras. En el análisis comparativo de los objetivos de cada país, se identifica un traslape de intereses nacionales, mezclados con los intereses regionales, donde los primeros aún tienen una influencia significativa en la estrategia y en el proceso de negociación.

Para México, la renegociación del TLCAN era un asunto deseable y previsible, pero la negociación basada en amenazas y tiempos perentorios no era el escenario óptimo para la modernización del TLCAN, y mucho menos que su renegociación se traslapara con el cambio de poder en el ejecutivo mexicano. De alguna forma, el TLCAN ya se había actualizado parcialmente por vía de la negociación del Acuerdo Transpacífico (TTP), ya especialistas como Gustavo Vega Cánovas habían señalado oportunamente que los temas centrales de la negociación del nuevo TLCAN se hallaban prescritos en el TTP ${ }^{5}$, así como en otros tratados firmados por los tres países de América del Norte. La experiencia previa de negociación del TTP podía marcar una hoja de ruta factible para el T-MEC, el problema sería la cantidad de temas y concesiones previas que el gobierno de Trump permitiría que sobrevivieran del TTP fallido. Para entender la estrategia que siguió México en las negociaciones del T-MEC y que influyó en los resultados obtenidos en las $\mathrm{R}$ de $\mathrm{O}$, es pertinente recordar las razones que en su momento tuvo tanto el gobierno mexicano como el canadiense para negociar el TTP, las cuales se vieron influidas por dos asuntos: a) el imperativo interés por defender su participación en el mercado de los Estados Unidos, y b) procurar reducir los efectos directos y secundarios que pudieran resultar de los cambios en la política económica y comercial propugnados desde los tiempos del gobierno demócrata de Barak Obama en Estados Unidos.

Desde el inicio de las negociaciones, el gobierno de México pretendió modernizar el acuerdo negociándolo a partir de una estrategia basada en 4 principios que servirían de guía durante todo el proceso: a) apoyar la competitividad del país en la región de América del Norte por medio de la liberalización de bienes y servicios, la desregulación, la expansión del acceso preferencial y libre a los mercados, y la atracción de la inversión extranjera directa; b) propiciar un acuerdo comercial con reglas más incluyentes y sustentable por medio de la agregación de reglas de última generación en materia laboral, ambiental, las dirigidas a las pequeñas y medianas empresas (PYMEs), las normas que faciliten el comercio y las reglas anticorrupción; c) procurar aprovechar las oportunidades ofrecidas por la nueva era tecnológica con la incorporación de normas sobre comercio electrónico, la propiedad intelectual, y las tecnologías financieras; y d) mantener un entorno comercial y de inversiones predecible fortaleciendo los mecanismos de solución de controversias y el establecimiento de regulaciones sobre compras gubernamentales, libre competencia y definiendo el papel de las empresas de propiedad estatal (Bahri, 2020).

Además, México dibujó en las negociaciones sus límites aceptables en el acuerdo, los cuales no deberían rebasarse. Estos límites básicamente manifestaban su oposición firme a las amenazas usadas por el gobierno de Trump como táctica de negociación, las cuales eran: la eliminación de los mecanismos de solución de controversias; el retroceso en el acceso a la contratación pública; la inclusión de una cláusula de extinción automática del acuerdo después de cinco años, las medidas comerciales correctivas basadas en las temporadas estacionales y los contingentes aplicados a la agricultura; retirar el acceso a los servicios de transporte transfronterizo, y particularmente importante para el tema que se aborda en este estudio, modificar las $\mathrm{R}$ de $\mathrm{O}$ y establecer

México, Nueva Zelanda, Perú, Singapur, Estados Unidos y Vietnam.
${ }^{5}$ El TTP fue firmado el 4 de febrero de 2016 por Australia, Brunei, Canadá, Chile, Japón, Malasia, 
restricciones comerciales cuantitativas.

Tanto para el gobierno de Canadá como para el de México, aceptar modificaciones a las $\mathrm{R}$ de $\mathrm{O}$ no era algo sujeto a negociación, pues ya desde la participación de ambos países en la creación del TTP, se había decidido que su redacción les iba a dar un acceso estratégico y privilegiado para alcanzar la meta de acumular origen, a partir de las $\mathrm{R}$ de $\mathrm{O}$ adecuadas a sus intereses que quedaron plasmadas en el propio TTP, lo cual era singularmente importante para industrias estratégicas ubicadas en ambos países. El gobierno de México había argumentado que iba a ser necesario previamente un examen exhaustivo de las $\mathrm{R}$ de $\mathrm{O}$. También expresó que incluir $\mathrm{R}$ de $\mathrm{O}$ específicas para cada uno de los países en el TLCAN sería "inaceptable" para México.

Además, hubo cierto escepticismo sobre si el nuevo gobierno de Andrés Manuel López Obrador apoyaría la renegociación del TLCAN, ya que él había expresado antes su apoyo a varias acciones proteccionistas como el aumento del contenido nacional en las cadenas de valor, el otorgamiento de subsidios al sector agrícola para lograr autosuficiencia alimentaria y un mayor uso de los mecanismos de defensa del comercio internacional. Por ello, existía el temor de que la ideología del gobierno, que por mucho tiempo había apoyado la liberalización comercial tuviera una transformación con el cambio de partido en el poder (Cruz Vargas, 2018).

Intereses y posición de Estados Unidos.

Para entender la posición de los EU en las negociaciones de las $\mathrm{R}$ de $\mathrm{O}$ se debe tener claro que para los gobiernos de Obama y de Trump, los acuerdos comerciales regionales y/o bilaterales representaron un plan B frente a la imposibilidad de continuar negociando temas comerciales a nivel multilateral en el seno de la OMC, fue bajo esta premisa que se abordó el fallido TTP como un medio para promover un régimen internacional de

6 Las críticas de Donald Trump se aceptaron por el descontento con la globalización, que proviene de un intenso sentimiento de marginación y olvido en algunos sectores, principalmente en la clase trabajadora, la cual votó por el candidato republicano.

7 "The US is seeking to strengthen rules of origin in order to "incentivize the sourcing of goods and materials from the United States and North America," as well as streamline associated administrative procedures and ensure their vigorous enforcement. It's part of Washington's broader "America First" agenda aimed at comercio e inversión con base en sus intereses regionales; y que desde entonces los EU fijaron sus objetivos de negociación para este acuerdo y para los próximos -incluyendo el T-MEC- del siguiente modo: 1) asegurar para EU el papel principal en la construcción del régimen económico internacional basado en sus principios unilaterales de librecambismo; 2) procurarse un papel económico preponderante en el comercio mundial particularmente ante el resurgimiento de China; 3) desarrollar un marco operativo para lograr una asociación económica más profunda con aliados que contribuyan a equilibrar el propio peso de China a nivel global y regional; 4) incentivar un proceso de reformas políticas, económicas y sociales en aquellos países emergentes que aún no se han liberalizado por completo (USTR, 2017).

Las negociaciones para la modernización del Tratado de Libre Comercio de América del Norte (TLCAN) iniciaron en el año 2017 como iniciativa del gobierno de los Estados Unidos por medio de su presidente Donald Trump, quién desde su campaña electoral prometió que revertiría el déficit que tenía la balanza comercial de su país con sus socios del TLCAN $^{6}$, amenazando con renunciar a cumplir el propio TLCAN, o renegociar los acuerdos comerciales tanto multilaterales, regionales $\mathrm{o}$ bilaterales firmados por los EU. Esta búsqueda por revertir el desequilibrio sería el principio negociador básico, tal y como lo dio a conocer el secretario de comercio Wilbur Ross cuando señaló que el propósito de EU era robustecer las $\mathrm{R}$ de $\mathrm{O}$. En una carta enviada al representante comercial de EU, se detallaron los objetivos para la renegociación del TLCAN, entre ellos el propósito de fortalecer las $\mathrm{R}$ de $\mathrm{O}$ sin poner obstáculos innecesarios a los flujos comerciales ${ }^{7}$. Según Ross un análisis del Departamento de Comercio (formulado a partir de los datos comerciales de valor agregado publicados recientemente por la OCDE), encontró que el contenido de EU en las importaciones automotrices de México disminuyó al $18.1 \%$ en 2011 a partir del $26.5 \%$ en $1995^{\circ}$. El contenido de EU en las

creating high-paying US manufacturing Jobs". https://policyoptions.irpp.org/fr/magazines/august2017/understanding-the-nafta-rules-of-originnegotiations/

${ }^{8}$ El contenido de valor agregado automotriz de países no miembros del TLCAN en las importaciones de automóviles mexicanos aumentó a 29.5 por ciento desde 13.2 por ciento. China ha experimentado el crecimiento más rápido en ese componente, al 5,8 por ciento en 2011 desde un escaso 0,3 por ciento en 1995 . 
importaciones automotrices de Canadá disminuyó al $26.4 \%$ a partir del $34.9 \%$ en el mismo período. Ross había criticado antes las R de O del TLCAN al considerarlas como una "puerta trasera" para que las autopartes chinas ingresasen a Estados Unidos a través de México y Canadá, por ello -argumentabalos beneficios del pacto comercial debían restringirse únicamente a sus miembros (Lawder, 2017).

Así pues, los objetivos de los EU en materia de $\mathrm{R}$ de $\mathrm{O}$ fueron: actualizar y reforzar las normas de origen, según fuese necesario, para garantizar que los beneficios del TLCAN se apliquen a los bienes a productos realmente elaborados en América del Norte y en EU en particular. Asegurar que las R de $\mathrm{O}$ incentiven el abasto de bienes e insumos de la región. Establecer procedimientos que agilicen la certificación y verificación de las normas de origen, y que fomenten una aplicación rigurosa. Promover la cooperación con los países del TLCAN para garantizar que los bienes que cumplen con las normas de origen reciban los beneficios del acuerdo, eviten la evasión de impuestos y combatan los delitos aduaneros. Tales objetivos encajarían en lo que llamamos el "área gris" de la integración, en otras palabras, objetivos nacionales de negociación que empujan tanto a la integración, o como imponer los objetivos nacionales económicos de EU por encima de los regionales.

\section{Intereses y posición de Canadá}

Estudios realizados por especialistas en el tema de las $\mathrm{R}$ de $\mathrm{O}$, han identificado que la mitad de las exportaciones comerciales de Canadá a los Estados Unidos entraron libres de aranceles buscando calificar para beneficiarse de las preferencias arancelarias del TLCAN. Otro 38\% no pagó aranceles, al ingresar bajo rubros libres de aranceles. En los casos en que las exportaciones canadienses enfrentaron un arancel NMF positivo, el uso del arancel preferencial del TLCAN estuvo cerca del $80 \%$ en general, y las tasas excedieron el 90\% para algunas de las principales exportaciones de bienes canadienses, como los automóviles.

A partir de lo anterior, se entiende que las negociaciones del T-MEC ofrecían a Canadá una gran oportunidad para flexibilizar las $\mathrm{R}$ de $\mathrm{O}$ al momento de aplicarlas, y hacerlas menos onerosas desde un punto de vista burocrático-administrativo. El gobierno canadiense tenía a su disposición la estrategia de presionar a los EU para simplificar la forma en que se calcula el contenido regional de un bien, de modo que el enfoque estuviera únicamente centrado en los componentes o materiales clave (a través de un método llamado "método de valorcontenido regional focalizado"). Ya el gobierno de Canadá había realizado mejoras al respecto en sus acuerdos comerciales negociados en el pasado reciente después del TLCAN, como sucedió en el TTP y en y el Acuerdo Económico y Comercial Integral Canadá-UE. Dentro del propio TTP se había acordado una solución para este problema, por lo que Canadá consideró que debía dársele continuidad en las negociaciones del nuevo TLCAN.

Por tanto, se esperaba que en la negociación de las $\mathrm{R}$ de $\mathrm{O}$, Canadá debía estar abierto a una propuesta razonable para garantizar que las reglas se aplicaran de manera efectiva, y al mismo tiempo asegurarse que los EU no se salieran con la suya en lo concerniente a introducir métodos agresivos de aplicación de las reglas, que no incluyesen protecciones adecuadas para los derechos de los productores, exportadores e importadores (como el derecho de notificación previa a las visitas de verificación de los funcionarios de aduanas).

Otra área en la que los negociadores canadienses iban a poner atención se trataba de la "acumulación trilateral", la cual se refiere a la posibilidad de los productores de obtener -sin restricciones- materiales y bienes libres de impuestos de alguno de los países del TLCAN. Esta posibilidad creada bajo el TLCAN había fomentado el crecimiento de las cadenas de suministro en toda América del Norte en muchas industrias, las cuales habían contribuido significativamente tanto al aumento de la competitividad de los productores norteamericanos como a los beneficios económicos de los que disfrutan las tres partes.

Así pues, durante las negociaciones del TLCAN, iba a ser necesario que tanto Canadá como México resistieran las demandas de EU de que los productos incorporen una cantidad específica de contenido estadounidense. Las cosas se habrían complicado para el comercio de la región, si las tres partes impusieran requisitos de contenido específicos para cada país, en lugar de para toda la región, porque entonces los productores tendrían dificultades para obtener insumos a precios competitivos en América del Norte. Su competitividad futura también se vería comprometida si, además de tener que afrontar las cargas administrativas y los costos de cumplir con tres conjuntos de requisitos de contenido específicos de cada país, también hubieran tenido que lidiar con $\mathrm{R}$ de $\mathrm{O}$ del TLCAN más restrictivas para sus productos (Reinch, 2021). 
Es así como Canadá y México debían esforzarse para procurar minimizar cualquier ajuste a las $\mathrm{R}$ de $\mathrm{O}$, así como detener cualquier intento de limitar o interrumpir la acumulación trilateral reconocida bajo las reglas del TLCAN dada la importancia de las cadenas de suministro de América del Norte. Canadá también debía procurar abordar sus prioridades, como las reglas aplicadas al petróleo crudo, y tomar la iniciativa para liberalizar las reglas para productos específicos o reducir los costos administrativos y la complejidad de las R de O del TLCAN. En resumen, los intereses económicos de Canadá exigían la liberalización de las reglas para reflejar la globalización de la producción que ha tenido lugar desde 1994.

Resultado final.

Si se toma en cuenta que ya existía un mecanismo para actualizar progresivamente las reglas dentro del TLCAN por medio del comité de las $\mathrm{R}$ de $\mathrm{O}$, sorprende que los EU hayan propuesto un cambio significativo en ellas para que quedase plasmado en el T-MEC actualizado. En el nuevo TMEC se aprecia un apuntalamiento de las $\mathrm{R}$ de $\mathrm{O}$ en aquellos sectores exitosos en donde la creación de las cadenas de valor regionales han dado lugar a sectores muy dinámicos como es el caso del automotriz, el texto del T-MEC abarca ahora normas de origen más estrictas pero también más transparentes en este ámbito, el cual además abarca una proporción significativa del comercio entre los países del TLCAN; pero ¿cuáles serán los costos reales derivados de la instrumentación de tales reglas? eso es un asunto por verse, considérese que durante las negociaciones del TLCAN como resultado de las presiones del gobierno de Canadá, y teniendo como antecedente el AUTOPAC firmado por los Estados Unidos y ese país en 1965, se buscó establecer $\mathrm{R}$ de $\mathrm{O}$ claras y fáciles de instrumentar a partir de la entrada en vigor del TLCAN en 1994. La preocupación de Canadá era que el contenido del valor regional aportado a los autos pudiera medirse sin dificultades, para lo cual se estableció una fórmula conocida como "costo neto" la cual se expresa a continuación:

$$
\mathrm{CVR}=\frac{\mathrm{CN}-\mathrm{VMN}}{\mathrm{CN}} \times 100
$$

En donde CVR representa el contenido de valor regional del auto, mientras que NC representa el costo neto del producto, y VMN el valor de los

${ }^{9}$ Sólo las empresas automotrices saben con precisión dónde se fabrica cada elemento de su producto y, por lo tanto, el alcance de los cambios que serían necesarios materiales que no son originarios de la región (Marise, 2020). Las partes en el acuerdo esperaban que con esta fórmula el costo neto calculado fuera más transparente y previsible debido a que incluía todos los costos de producción excepto los negociados expresamente entre las partes (como el marketing, promoción de ventas, costos del servicio posventa y similares), y además las empresas privadas se mostraron muy satisfechas con el acuerdo logrado.

Con el T-MEC las nuevas normas cambiarán el juego, pues la parte del valor total que se debe fabricar de un auto en América del Norte para obtener los beneficios del TLCAN se eleva a $75 \%$ con respecto del actual $62.5 \%$ para automóviles y autopartes, con algunas excepciones, como los camiones pesados, para los cuales el requisito es del $70 \%$. Estos nuevos requisitos se elaboraron para escalonarse en más de cinco años. En el TLCAN el $62.5 \%$, permitía los beneficios del libre comercio para bienes con un valor significativo producido en China, por ejemplo, y otros países no norteamericanos, pero imponiendo topes al mismo. También se incluye una innovación que requerirá que los trabajadores que ganan US $\$ 16$ por hora o más, produzcan el $40 \%$ del valor de los automóviles, y el $45 \%$ del valor de los camiones ligeros para que el producto terminado califique para un tratamiento aduanero libre de impuestos, en todos estos casos la norma es clara y no deja mucho espacio a libres interpretaciones.

Este requisito de Contenido de Valor Laboral (LVC por sus siglas en inglés) es toda una aportación singular del T-MEC que subraya un concepto importante que vincula el acceso al mercado comercial con los niveles salariales, y sienta un precedente importante para los pactos comerciales del futuro (sobre todo, aquellos firmados entre países con grandes asimetrías económicas entre ellos). Pero hasta la fecha ha sido difícil calcular los efectos prácticos del LVC acerca de dónde se ubicarán los trabajos de ensamblaje y piezas de automóviles, y sobre los niveles salariales que terminarán prevaleciendo en los Estados Unidos, México o Canadá9.

Si bien las R de $\mathrm{O}$ aplicadas al sector automotriz han recibido más atención, el texto del T-MEC tiene más $\mathrm{R}$ de $\mathrm{O}$ altas en otros capítulos, y en sectores muy distintos al automotriz, "el texto negociado

para cumplir con el porcentaje requerido de producción de los trabajadores a los que se les pagan US\$16 o más. 
mejora el TLCAN original en una serie de formas que deberían aumentar la producción y el empleo en América del Norte" (Feery, 2018). Ejemplos de tales mejoras son la eliminación de metodologías para calcular el valor de los insumos en un producto, que aportan la minimización de la contabilidad del contenido extranjero, reglas más claras sobre el origen del acero y el aluminio utilizados para fabricar productos, y un cambio en el enfoque en la determinación del "origen" de un bien, considerando el valor al momento de ingresar a un país, en lugar de si se producen cambios finales o un ensamblaje del mismo bien.

En el T-MEC se pactó una regla de minimis del $10 \%$ para las mercancías que contienen materiales no originarios que no se someten a un cambio aplicable en la clasificación arancelaria. Bajo esta regla, si el valor de los materiales no originarios que no se someten a un cambio aplicable en la clasificación arancelaria no es mayor al 10\% del valor de transacción del producto terminado o su costo total, el bien se considera originario Si un producto tiene un $10 \%$ o menos de materiales no originarios que no se someten a un cambio arancelario aplicable, el umbral de minimis puede utilizarse para lograr un trato arancelario preferencial. Sin embargo, si un producto tiene un $12 \%$ de materiales no originarios que no se someten a ese cambio, la regla de minimis no se aplica y no se puede utilizar para lograr el carácter originario. Finalmente, hay algunas excepciones a la regla de minimis. La mayoría de estas excepciones se refieren casi en su totalidad a productos agrícolas y alimenticios, aunque algunas excepciones también se aplican a los productos minerales. En el Anexo 4-A de las $\mathrm{R}$ de $\mathrm{O}$ del T-MEC se puede encontrar una lista completa de excepciones a la regla de minimis. Los productos textiles también pueden estar sujetos a reglas de minimis especializadas, de conformidad con los Artículos 4.12 y el Capítulo 6 del T-MEC.

Con el nuevo T-MEC no existe un certificado de origen oficial; sin embargo, todas las certificaciones deben contener datos básicosespecíficos relacionados con la transacción ${ }^{10}$. Las certificaciones y la información utilizada que respalda la certificación deben conservarse durante

${ }^{10}$ Una certificación de origen que sea la base para una solicitud de trato arancelario preferencial bajo el USMCA deberá incluir: a. Indicación de si el certificador es el Exportador, Productor o Importador; segundo. Nombre, cargo, dirección (incluido el país), dirección de correo electrónico y número de teléfono del certificador; C. Nombre del exportador, dirección (incluido el país) e
5 años. Esto abarca registros y documentación de respaldo relacionada con la importación, todos los registros y documentos de respaldo relacionados con el origen del bien (p. ej., registros de producción, registros de abasto, valor y procesamiento de los materiales no originarios, etc.), y los registros y la documentación de respaldo necesarios para demostrar el cumplimiento del requisito del T-MEC. Estados Unidos, México o Canadá pueden aplicar medidas según las circunstancias lo justifiquen cuando una entidad certificadora dentro de su territorio ofrece una certificación para bienes que no cumplen con los requisitos del T-MEC, o para los cuales el certificador no ha mantenido la documentación de respaldo adecuada. $\mathrm{Si}$ las autoridades aduaneras del país de importación determinan que el importador hizo declaraciones falsas o no fundamentadas para obtener una preferencia dentro del T-MEC, las reclamaciones serán denegadas y el importador deberá pagar los derechos después del hecho. Las sanciones también pueden ser evaluadas en determinados casos. El establecimiento de procesos para una organización precisa y retención de documentos facilitará cualquier posible auditoría y ayudar a las empresas a demostrar fácilmente el cumplimiento si es necesario.

\section{CONCLUSIONES.}

La teoría de la integración económica anticipaba que, después del relativo éxito alcanzado en América del norte con la entrada en vigor de un área de libre comercio que duró 25 años (el TLCAN), el siguiente paso por dar entre los tres países era la construcción de una unión aduanera, pero debido a la naturaleza distributiva de las negociaciones y a los obstáculos citados en esta investigación, lejos de acercarnos a la unión aduanera el T-MEC está por instrumentar un mecanismo más o menos complejo de $\mathrm{R}$ de $\mathrm{O}$, como sustituto de ella, que podría elevar los costos de transacción con respecto al tipo de facilitación del comercio planteada antiguamente por el TLCAN, como se procuró demostrar con lo que podría suceder en el sector automotriz de la región. Paradójicamente, estas $\mathrm{R}$ de $\mathrm{O}$ pueden convertirse en un referente para otros acuerdos comerciales negociados en distintas regiones del mundo,

información de contacto, nombre del productor, dirección (incluido el país) e información de contacto, y el nombre del importador, la dirección (incluido el país) e Información del contacto y Firma del certificador Muchas empresas de transporte y transporte tienen formularios de certificación accesibles en línea que se pueden completar y utilizar para certificar bienes T-MEC. 
particularmente aquellos en donde existan asimetrías en las capacidades económicas de los países.

Redactar reglas más estrictas podría conducir negativamente a un contenido regional más bajo en los productos finales y perturbar las cadenas de suministro regionales, pues cada vez más importadores evitan las preferencias del TLCAN debido a las costosas $\mathrm{R}$ de $\mathrm{O}$ y prefieren comerciar con aranceles NMF. Una vez que los bienes se importan a través de medios no preferenciales, las $\mathrm{R}$ de $\mathrm{O}$ se vuelven irrelevantes, por lo que es probable que el contenido regional disminuya. Incluso si aumenta el contenido del TLCAN, las $\mathrm{R}$ de $\mathrm{O}$ hacen poco por aumentar la participación del contenido estadounidense. Si el objetivo es más contenido estadounidense, las normas de origen no son un medio eficaz. Las PyMEs se ven especialmente desfavorecidas por las engorrosas normas de origen porque los costos burocráticos de hacer negocios representan una parte mayor de sus costos totales.

\section{REFERENCIAS}

Bahri, A., \& Lugo, M. (2020). Trumping Capacity Gap with Negotiation Strategies: The Mexican USMCA Negotiation Experience. Journal of International Economic Law, 23(1), 1-23.

Bergsten, Fred (2019) A Path Forward for NAFTA. 2019, July 09. Consultado el 23 de noviembre 2020, en https://www.piie.com/publications/piie-briefings/path-forward-nafta,

p.

121. Cadot, Olivier, and Jaime de Melo. 2007. Why OECD Countries Should Reform Rules of Origin. Lausanne, Switzerland: University of Lausanne.

Cadot, Olivier, Jaime de Melo, Antoni Estevadeordal, Akiko Suwa-Eisenmann, y Bolormaa Tumurchudur. (2002). Assessing the Effect of NAFTA's Rules of Origin. Paper Estudio Comparativo sobre las Normas de Origen Preferenciales. (2018). Consultado en http://www.wcoomd.org//media/wco/public/es/pdf/topics/origin/instruments-and-tools/reference-material/170130-c-_spcomparative-study-on-pref_roo_-master-file_final-23_06_2017.pdf

Falvey, Rod, y Geoff Reed. (1998). Economic Effects of Rules of Origin. Weltwirtschaftliches Archiv 134, no. 2: 209-29.

Gregory Spak Francisco de Rosenzweig Dean A. Barclay Matt Solomon Brian Picone. (2018). Overview of Chapter 4 (Rules of Origin) of the US-Mexico-Canada Trade Agreement. Consultado el 20 de enero, en https://www.whitecase.com/publications/alert/overview-chapter-4-rules-origin-us-mexico-canadatrade-agreement.

Senger, S. (2018). Understanding the NAFTA rules of origin. Consultado el 1 de marzo 2021, en https://www.shippingsolutions.com/blog/nafta-rules-of-origin-part-1

Ju, Jiandon, y Kala Krishna. (2005). Firm Behaviour and Market Access in a Free Trade Area with Rules of Origin. Canadian Journal of Economics. 38, no. 1: 290-308

Krishna, Kala. (2005). Understanding Rules of Origin. NBER Working Paper 11150. Cambridge, MA: National Bureau of Economic Research. 
Moroz, Andrew. (2017). Navigating the Maze: Canada, Rules of Origin and the Trans-Pacific Partnership (and Two Tales of Supply Chains). In Redesigning Canadian Trade Policies for New Global Realities, ed. Ari Van Assche, Robert Wolfe, and Stephen Tapp. Montreal, Canada: Institute for Research on Public Policy.

Puyana, Alicia (2014) La política comercial de México. El legado de los veinte años del TLCAN. En Arturo Oropeza, “TLCAN, 20 años. ¿Celebración, desencanto o replanteamiento?”, Instituto de Investigaciones Jurídicas UNAM.

Reinch, W. A. (2021). The Impact of Rules of Origin on Supply Chains: USMCA's Auto Rules as a Case Study. Consultado en https://www.csis.org/analysis/impact-rules-origin-supply-chains-usmcas-autorules-case-study

Vega, Gustavo. (2020). La integración económica en América del Norte ante la ... consultado 23 julio 2020, en http://www.scielo.org.mx/pdf/fi/v57n4/0185-013X-fi-57-04-00781.pdf

Vega, Gustavo. (2020). "La facilitación del comercio en la renegociación del TLCAN y en el TMEC" en Contreras, Oscar. f. et. al. La reestructuracion de norteamerica a traves del libre comercio del TLCAN al TMEC: El Colegio de México.

Viner, Jacob (1950) "The Customs Union Issue", capítulo IV (Londres: Stevens, 1950), reproducido íntegramente en Miroslav Jovanovic, International Economic Integration. Critical Perspectives on the World Economy, vol II

UNCTAD (2013), World Investment Report 2013. Global Value Chains: Investment and Trade for Development, Nueva York y Ginebra, United Nations, 2013, p. xxii.

USTR (2017) USTR Releases Updated NAFTA Negotiating Objectives https://ustr.gov/about-us/policyoffices/press-office/press-releases/2017/november/ustr-releases-updated-nafta

WORLD TRADE ORGANIZATION. (2021). Agreement on rules of origin. Consultado el 28 de enero 2021, en https://www.wto.org/english/tratop_e/roi_e/roi_info_e.htm 
